\title{
MULTIPLET SHORTENING IN Osp(N,4)*
}

\author{
Daniel Z. FREEDMAN \\ Department of Mathematics, Massachusetts Institute of Technology, \\ Cambridge, Massachusetts 02139, USA
}

Hermann NICOLAI

TH Division, CERN, 1211 Geneva 23, Switzerland

Received 15 September 1983

\begin{abstract}
We describe a new type of multiplet shortening in $\operatorname{Osp}(N, 4)$ which resolves a long-standing puzzle in Kaluza-Klein supergravities. Multiplet shortening implies quantization of mass eigenvalues in units of the inverse AdS radius. While detailed proofs are presented only for $N=3$, we discuss implications for $N=8$ supergravity and derive the $\mathrm{SO}(8)$ assignments of all higher modes on the round seven-sphere.
\end{abstract}

\section{Introduction}

The work described in this paper is largely mathematical but the motivation comes from the following puzzle in spontaneously compactified supergravity theories. The 11-dimensional supergravity [1] theory has a classical solution with background metric $(\mathrm{AdS})_{4} \times \mathrm{S}_{7}$, full $N=8$ supersymmetry, and $\mathrm{SO}(8)$ internal symmetry $[2,3]$. The massless excitations are known to constitute a standard $N=8$ representation with maximum spin $2[4,3]$. There is also an infinite set of massive excitations with maximum spin 2 . Relatively little is known about them, but it is clear that they should form massive representations of $N=8$ supersymmetry and maintain $\mathrm{SO}(8)$ invariance since the vacuum state has these symmetries. In Poincare supersymmetry massive $N=8$ representations require either $s_{\max }=4$ with no central charges or $s_{\max }=2$ with central charges that break $\mathrm{SO}(8)$ [5]. Clearly, neither has the correct structure. Of course, in the anti-de Sitter spacetime of compactified supergravity the relevant superalgebra is $\operatorname{Osp}(N, 4)$, not Poincaré supersymmetry. Therefore a separate study of the massive representations of $\operatorname{Osp}(N, 4)$ is required to understand the higher modes.

We report here recent results on the structure of massive representations of $\operatorname{Osp}(N, 4)$ which indicate that there is a new type of multiplet shortening due to the

\footnotetext{
* Work supported in part by the National Science Foundation Grant 81-07394.
} 
internal symmetry generators in the algebra. This resembles the shortening due to central charges in Poincaré supersymmetry, but here the $\operatorname{SO}(N)$ automorphism group is maintained. To minimize algebraic complexity representations are explicitly constructed only for $N=3$, and we show that $\operatorname{Osp}(3,4)$ has a series of short representations with maximum spin 1 whereas the typical long representations require at least spin $\frac{3}{2}$. The analysis is considerably more complicated than for the Poincaré supersymmetry algebra, but it is facilitated by the following two basic observations.

(i) Any irreducible representation of $\operatorname{Osp}(N, 4)$ is decomposable in terms of the Osp $(1,4)$ multiplets which have been given in $[6]$.

(ii) In the limit where the radius of anti-de Sitter space becomes infinite and the $\operatorname{Osp}(N, 4)$ algebra contracts to the $N$-extended Poincare superalgebra, all irreducible representations of $\operatorname{Osp}(N, 4)$ become massless representations of Poincaré supersymmetry. This implies that all $\operatorname{Osp}(N, 4)$ multiplets can be obtained from products of (possibly reducible) representations of $\mathrm{SO}(N)$ with massless multiplets of Poincaré supersymmetry.

After giving the proof for $N=3$, we extract a simple procedure which correctly accounts for the spin and $S O(3)$ structure of the short representations. A natural generalization of this procedure to $N=8$ is conjectured. It appears to describe the short representations with $s_{\max }=2$ which are required in 11-dimensional supergravity. As a consequence, we derive the $\operatorname{SO}(8)$ assignments of all the massive modes on $\mathrm{S}_{7}$ in terms of $\mathrm{SO}(8)$ Dynkin labels.

Readers should note that the finite-dimensional representations of $\operatorname{Osp}(N, 4)$ are completely known [7]. However, it is the infinite dimensional representations which describe particles in supersymmetric field theories. Here there is a complete treatment only of the simplest case, $N=1$, in [6]. The general construction of unitary irreducible representations of non-compact supergroups has been studied in ref. [8], but the phenomenon of multiplet shortening has not been previously discussed.

\section{Known structure of irreducible representations}

For the convenience of the reader, we briefly review here what is known about irreducible representations of supersymmetry. For Poincaré supersymmetry, their structure is well-known [5]. There are massless representations with $2^{N}$ helicity states and helicity at least up to $\frac{1}{4} N$ for even $N$ or $\frac{1}{4}(N+1)$ for odd $N$, and there are long massive representations without central charges with at least $2^{2 N}$ helicity states and spins at least up to $\frac{1}{2} N$. With central charges there are short representations with the same spin limits as in the massless case. The general massless Poincare representation with a non-trivial Fock vacuum acted on by $2^{N}$ creation operators plays an important role in our work. (See sects. 9, 10).

It is perhaps less widely appreciated that the particle representations of $\operatorname{Osp}(1,4)$ are also completely known; they have been constructed by Heidenreich [5]. They 
decompose into positive energy representations of the covering group of $\operatorname{SO}(3,2) \sim$ $\mathrm{Sp}(4)$. These particle representations and their relation to wave equations in antide Sitter space were studied by Fronsdal [9]. A representation is denoted by $D\left(E_{0}, s\right)$ where $E_{0}$ is the lowest energy eigenvalue which occurs and $s$ is the total angular momentum quantum number of the lowest energy state. The representation is unitary provided that $E_{0} \geqslant s+1$ for $s=1, \frac{3}{2}, \ldots$ and $E_{0} \geqslant s+\frac{1}{2}$ for $s=0, \frac{1}{2}$. The representations are all infinite dimensional and weight diagrams for $s=0, \frac{1}{2}$, and 1 are shown in fig. 1. It is important that the notion of particle mass be interpreted in the context of anti-de Sitter space. A particle is massless if $E_{0}=(s+1)$ for $s=\frac{1}{2}, 1, \ldots$ or $E_{0}=1$ or 2 for $s=0$. It is known [9] that the corresponding wave equations are conformal invariant for $s=0, \frac{1}{2}$ and 1 and gauge invariant for $s \geqslant 1$.

Note that the vector weight diagram contains a set of scalar states (denoted by $x$ in fig. 1) which mix with the other states for $E_{0}>2$ but decouple for $E_{0}=2$. This corresponds to the decreased number of states which occur in the presence of gauge invariance and is suggestive of the Higgs mechanism. In the limit that the radius of the anti-de Sitter space becomes infinite with $E_{0}$ fixed, all representations approach massless representations of the Poincaré group. For $s=1$ and $E_{0}>2$, this WignerInönü contraction gives both helicity-1 and helicity-0 states.

Heidenreich found several types of representations of $\operatorname{Osp}(1,4)$, and we now give their $\operatorname{SO}(3,2)$ decomposition in a designation convenient for our purposes.

Type A: Wess-Zumino representations: $E_{0}>\frac{1}{2}$

$$
D\left(E_{0}, 0\right) \oplus D\left(E_{0}+\frac{1}{2}, \frac{1}{2}\right) \oplus D\left(E_{0}+1,0\right)
$$
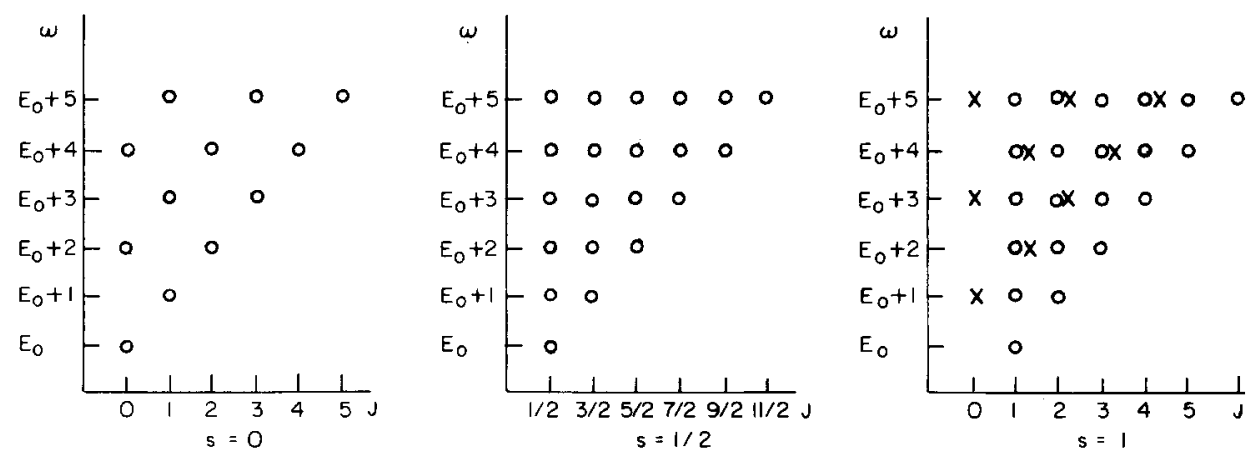

Fig. 1. Weight diagram of the $S O(3,2)$ representations $D\left(E_{0}, s\right)$ for $s=0, \frac{1}{2}, 1$. Each dot or cross indicates a rotational multiplet of $2 J+1$ states. Each representation is infinite dimensional so that the indicated patterns actually extend upward without bound. The crosses in the $s=1$ weight diagram indicate states of the scalar representation $D\left(E_{0}+1,0\right)$. The states are coupled with the others for $E_{0}>2$ but decouple for $E_{0}=2$ which corresponds to a massless gauge invariant vector field. See the text and [9] for further information on the particle representations of $\mathrm{SO}(3,2)$. 
Type B: Massive higher-spin representations: $E_{0}>s+1, s=\frac{1}{2}, 1, \ldots$

$$
D\left(E_{0}, s\right) \oplus D\left(E_{0}+\frac{1}{2}, s+\frac{1}{2}\right) \oplus D\left(E_{0}+\frac{1}{2}, s-\frac{1}{2}\right) \oplus D\left(E_{0}+1, s\right) .
$$

Type C: Massless higher-spin representations: $s=\frac{1}{2}, 1, \frac{3}{2}, \ldots$

$$
D(s+1, s) \oplus D\left(s+\frac{3}{2}, s+\frac{1}{2}\right) .
$$

The free supersymmetric field theories of type A and type C $\left(s=\frac{1}{2}\right)$ representations have been discussed both in superspace $[10,11]$ and component $[11,12]$ formulations. The field theory of the type $\mathrm{B}\left(s=\frac{1}{2}\right)$ representation has also been discussed recently [13]. For completeness we note that Heidenreich also found an $\operatorname{Osp}(1,4)$ representation involving Dirac singletons which we omit here because it does not appear to occur in standard field theory.

Very little is known about the unitary, irreducible representations of $\operatorname{Osp}(N, 4)$ for $N>1$. The structure of massless representations was inferred in [12] by using the type $\mathrm{C}$ and type $\mathrm{A}\left(E_{0}=1\right), N=1$ representations as building blocks for higher $N$. The same pattern as massless representations of Poincaré supersymmetry was found, viz. one $\mathrm{SO}(3,2)$ representation $D(s+1, s), N$ representations $D\left(s+\frac{1}{2}, s-\frac{1}{2}\right)$, etc.

Thus there is a perfect correspondence between all known $\operatorname{Osp}(N, 4)$ representations and those of Poincare supersymmetry and therefore no answer yet to the question of what representations describe the higher modes of compactified supergravity. In particular the known results give no hints of any new type of multiplet shortening.

\section{The $\operatorname{Osp}(N, 4)$ superalgebras}

The even elements of $\operatorname{Osp}(N, 4)$ consist of 10 hermitian $\operatorname{SO}(3,2)$ generators $M_{A B}=-M_{B A}$, where $A, B=0,1, \ldots, 4$, and $\frac{1}{2} N(N-1)$ hermitian $\operatorname{SO}(N)$ generators $T^{i j}=-T^{j i}$, where $i, j=1, \ldots, N$. The odd elements are $4 N$ Majorana spinor charges $Q_{\alpha}^{i}$ where $\alpha=1, \ldots, 4$ is a Dirac index. The structure relations of the superalgebra are,

$$
\begin{gathered}
\left\{Q_{\alpha}^{i}, \bar{Q}_{\beta}^{j}\right\}=i \delta^{i j}\left(l^{A B}\right)_{\alpha \beta} M_{A B}+i \delta_{\alpha \beta} T^{i j}, \\
l^{a 4}=-\frac{1}{2} i \gamma^{a}, \quad a=0,1,2,3, \\
l^{a b}=\frac{1}{4}\left[\gamma^{a}, \gamma^{b}\right]=\sigma^{a b}, \\
{\left[M_{A B}, Q_{\alpha}^{i}\right]=-i\left(l_{A B}\right)_{\alpha \beta} Q_{\beta}^{i},} \\
{\left[T^{i j}, Q_{\alpha}^{k}\right]=-i\left(\delta^{j k} Q_{\alpha}^{i}-\delta^{i k} Q_{\alpha}^{i}\right),} \\
{\left[M_{A B}, M_{C D}\right]=i\left(\eta_{B C} M_{A D}-\eta_{A C} M_{B D}-\eta_{B D} M_{A C}+\eta_{A D} M_{B C}\right),} \\
\eta_{A B}=(+,-,-,-,+), \\
{\left[T^{i j}, T^{k l}\right]=-i\left(\delta^{j k} T^{i l}-\delta^{i k} T^{j l}-\delta^{\prime \prime} T^{i k}+\delta^{i l} T^{j k}\right) .}
\end{gathered}
$$


Eq. (3) implies that the spinor charges transform in the $N$-dimensional vector representation of $\mathrm{SO}(N)^{\star}$. Later when we specialize to $N=3$, it is convenient to define $T^{i j}=\varepsilon^{i j k} T^{k}$; then (3) and (5) become

$$
\begin{aligned}
& {\left[T^{i}, Q_{\alpha}^{j}\right]=i \varepsilon^{i j k} Q_{\alpha}^{k},} \\
& {\left[T^{i}, T^{j}\right]=i \varepsilon^{i j k} T^{k}, \quad i, j \ldots=1,2,3 .}
\end{aligned}
$$

We will use the following $\gamma$-matrix representation

$$
\gamma^{0}=\left(\begin{array}{rr}
1 & 0 \\
0 & -1
\end{array}\right), \quad \gamma^{i}=\left(\begin{array}{cc}
0 & \sigma^{i} \\
-\sigma^{i} & 0
\end{array}\right), \quad C=i \gamma^{0} \gamma^{2}
$$

The Majorana spinor $Q_{\alpha}^{i}$ can then be parameterized as

$$
Q_{\alpha}^{i}=\left(\begin{array}{c}
a_{\alpha}^{i} \\
\varepsilon_{\alpha \beta} \bar{a}_{\beta}^{i}
\end{array}\right), \quad \varepsilon=\left(\begin{array}{rr}
0 & 1 \\
-1 & 0
\end{array}\right),
$$

where $\bar{a}_{\alpha}^{i}$ is the adjoint of $a_{\alpha}^{i}$. Since $M_{04}$ is the energy operator and $M_{i j}=\varepsilon_{i j k} J_{k}$ are rotation generators, (2) implies that

$$
\begin{aligned}
{\left[M_{04}, a_{\alpha}^{i}\right] } & =-\frac{1}{2} a_{\alpha}^{i}, \\
{\left[M_{04}, \bar{\alpha}_{\alpha}^{i}\right] } & =\frac{1}{2} \bar{a}_{\alpha}^{i}, \\
{\left[J_{k}, a_{\alpha}^{i}\right] } & =-\frac{1}{2}\left(\sigma_{k}\right)_{\alpha \beta} a_{\beta}^{j}, \\
{\left[J_{k}, \bar{a}_{\alpha}^{i}\right] } & =\frac{1}{2} \bar{a}_{\beta}^{i}\left(\sigma_{k}\right)_{\beta \alpha} .
\end{aligned}
$$

Thus $\bar{a}_{\alpha}^{i}$ and $a_{\alpha}^{i}$, respectively, raise and lower energy by $\frac{1}{2}$ unit, and $\bar{a}_{\alpha}^{i}$ is a standard irreducible spinor operator with respect to spatial rotations.

Finally we write out (1) in matrix form as

$$
\begin{aligned}
& \left(\begin{array}{cc}
\left\{a_{\alpha}^{i}, \bar{a}_{\beta}^{j}\right\} & \left\{a_{\alpha}^{i}, a_{\gamma}^{j}\right\} \varepsilon_{\gamma \beta} \\
\varepsilon_{\alpha \gamma}\left\{\bar{a}_{\gamma}^{i}, \bar{a}_{\beta}^{j}\right\} & \varepsilon_{\alpha \gamma}\left\{\bar{a}_{\gamma}^{i}, a_{\delta}^{j}\right\} \varepsilon_{\delta \beta}
\end{array}\right) \\
& =\left(\begin{array}{cc}
\delta^{i j}\left(M_{04}+\sigma^{k} J_{k}\right)_{\alpha \beta}+i T^{i j} \delta_{\alpha \beta} & \delta^{i j} \sigma_{\alpha \beta}^{k}\left(i M_{0 k}+M_{k 4}\right) \\
\delta^{i j} \sigma_{\alpha \beta}^{k}\left(i M_{0 k}-M_{k a}\right) & \delta^{i j}\left(-M_{04}+\sigma^{k} J_{k}\right)_{\alpha \beta}+i T^{i j} \delta_{\alpha \beta}
\end{array}\right) .
\end{aligned}
$$

* The case $N=8$ is special in that there exist three inequivalent 8-dimensional representations. The assignment of the spinor charges to any of these is a matter of conventions. 
This shows that anti-commutators of the odd elements (or, more generally, even products of the odd elements which are symmetric under interchange of index pairs

$$
\stackrel{i}{\alpha}, \ldots
$$

are related to even elements of the $\operatorname{Osp}(N, 4)$ algebra (or products thereof) or vanish for particular index values.

\section{Strategy of construction of representations}

The technique used here is a generalization of that of [6]. It is valid for any $N$, but it is convenient for notational reasons to specialize to $N=3$ and to use the standard notation $J, M$ to designate eigenvalues of the isospin Casimir operator $T^{i} T^{i}$ and of $T^{3}$. A representation of the even subalgebra $\operatorname{SO}(3,2) \times \operatorname{SO}(3)$ is denoted by $D\left(E_{0}, s, J\right)$ where, as before, $E_{0}$ is the lowest-energy eigenvalue in the multiplet and $s$ denotes the total angular momentum of the lowest energy state. $J$ is the common total isospin of all states in the representation. A typical state of the representation is written as $\left|\left(E_{0}, s, J\right), \omega, j, m, M\right\rangle$. Here, $\omega$ denotes the eigenvalue of the energy operator $M_{04}$, which differs from $E_{0}$ by an integer, $j$ and $m$ designate eigenvalues of the Casimir operator $J_{k} J_{k}$ and $J_{3}$ of the subalgebra of spatial rotations, and $M$ the eigenvalue of $T^{3}$.

For positive energy representations of $O s p(3,4)$ there must exist a unique vacuum multiplet of $(2 s+1)(2 J+1)$ states $\left|\left(E_{0}, s, J\right), E_{0}, s, m, J, M\right\rangle=|\mathrm{vac}, m, M\rangle$ with the property that they are annihilated by all energy lowering operators $a_{\alpha}^{i}$ :

$$
a_{\alpha}^{i}|\operatorname{vac}, m, M\rangle=0 \text {. }
$$

According to (11) all operator bilinears of the form $a_{\alpha}^{i} \bar{a}_{\beta}^{j}$ act on the vacuum as combinations of the operators $M_{04}, J_{k}$ and $T^{i}$ and therefore leave the vacuum multiplet invariant. Indeed $J_{k}$ and $T^{i}$ have the standard action of $\mathrm{SU}(2) \times \mathrm{SO}(3)$ generators on any state. The $\operatorname{SO}(3,2)$ energy boosts $M_{k}^{+}=i M_{0 k}-M_{k 4}$ are determined from (11) as

$$
\delta^{i j} M_{k}^{+}=\frac{1}{2}\left(\sigma_{k} \varepsilon\right)_{\alpha \beta}\left\{\bar{a}_{\alpha}^{i}, \bar{a}_{\beta}^{j}\right\}
$$

This means that the bilinears $\left\{\bar{a}_{\alpha}^{i}, \bar{a}_{\beta}^{j}\right\}$ either annihilate the vacuum states if $i \neq j$ or, if $i=j$, they give a linear combination of states $\left|\left(E_{0}, s, J\right), E_{0}+1, j, m^{\prime}, M\right\rangle$ of the first excited level of the $\mathrm{SO}(3,2)$ representation in which the vacuum lies. Note that in general one may have $j=(s+1), s$, or $(s-1)$ as in fig. 1. Further application of the bilinears $\left\{\bar{a}_{\alpha}^{i} \bar{a}_{\beta}^{j}\right\}$ simply moves us higher in energy among the states of the representation $D\left(E_{0}, s, J\right)$ but leaves the isospin content of the state unchanged. 
We now introduce the set $\overline{\mathbf{B}}$ which consists of all combinations of products of the operators $\bar{a}_{\alpha}^{i}$ which are antisymmetric under interchange of the index-pairs $\left(\begin{array}{l}i \\ \alpha\end{array}\right)$. It is advantageous to divide $\overline{\mathrm{B}}$ into subsets $\overline{\mathrm{B}}_{0}, \ldots, \overline{\mathrm{B}}_{2 N}$ where $\overline{\mathrm{B}}_{n}$ contains products of $n$ operators $\bar{a}_{\alpha}^{i}$. Thus, we have

$$
\overline{\mathbf{B}}=\overline{\mathbf{B}}_{0} \cup \cdots \cup \overline{\mathbf{B}}_{2 N},
$$

with

$$
\begin{array}{ll}
\overline{\mathrm{B}}_{0} & =\{1\}, \quad \overline{\mathrm{B}}_{1}=\left\{\bar{a}_{\alpha}^{i}\right\}, \quad \overline{\mathrm{B}}_{2}=\left\{\left[\bar{a}_{\alpha}^{i}, \bar{a}_{\beta}^{j}\right]\right\}, \\
\overline{\mathrm{B}}_{3} & =\left\{\sum_{\text {perm }} \pm \bar{a}_{\alpha}^{i} \bar{a}_{\beta}^{j} \bar{a}_{\gamma}^{k}\right\},
\end{array}
$$

etc.

From (15), it is evident that $\overline{\mathrm{B}}_{n}$ contains $\left(\begin{array}{c}2 N \\ n\end{array}\right)$ operators and therefore $\overline{\mathrm{B}}$ contains $2^{2 N}$ operators. For $N=3$, which is the case on which we will concentrate from now on, there are 64 such operators. By construction, the symmetric combinations and the set $\overline{\mathrm{B}}$ are mutually exclusive, and it is obvious that any product of operators $\bar{a}_{\alpha}^{i}$ can be expressed as a sum of products of even elements of the $\operatorname{Osp}(N, 4)$ algebra and elements of $\bar{B}$.

When an operator of $\overline{\mathbf{B}}_{n}$ acts on a vacuum state it typically gives a linear combination of lowest weight states of new $\mathrm{SO}(3,2) \times \mathrm{SO}(3)$ representations

$$
D\left(E_{0}+\frac{1}{2} n, s^{\prime}, J^{\prime}\right),
$$

where $s^{\prime}$ and $J^{\prime}$ are any of the several spin and isospin values which are obtained by Clebsch-Gordan addition of $s$ and $J$ of the vacuum with the spin and isospin carried by the operator of $\bar{B}_{n}$. In addition, as we will see in detail later, there may be admixtures of states obtained by applying energy boosts to lowest weight states of representations $D\left(E+\frac{1}{2} m, S^{\prime \prime}, J^{\prime}\right)$ for $m<n$ if such states are generated by operators of $\overline{\mathrm{B}}_{m}$ acting on the vacuum.

We now define an infinite-dimensional vector space $V$ as the span of all vectors of the form

$$
\left(M_{1}^{+}\right)^{n_{1}}\left(M_{2}^{+}\right)^{n_{2}}\left(M_{3}^{+}\right)^{n_{3}} \overline{\mathrm{B}}|\mathrm{vac}, m, M\rangle \text {, }
$$

where $n_{i}$ are any non-negative integers and where any operator in the set $\overline{\mathrm{B}}$ can appear in the last position. A unitary representation of $O s p(3,4)$ can be defined on $V$ by imposing a Hilbert space structure. The vacuum multiplet is assumed to be orthonormal. The norms of other states in $\mathrm{V}$ are then calculated, in order of 
increasing energy, by moving $a$ operators to the right of $\bar{a}$ operators using $(2,3,11,12)$. The requirement of nonnegative norm will impose restrictions on the quantum numbers $E_{0}, s, J$ of the vacuum. The typical long representations of $\operatorname{Osp}(3,4)$ contain all $\mathrm{SO}(3,2) \times \mathrm{SO}(3)$ representations $D\left(E_{0}+\frac{1}{2} n, s^{\prime}, J^{\prime}\right)$ with values of $s^{\prime}, J^{\prime}$ that occur in the Clebsch-Gordan decomposition of operators of $\overline{\mathrm{B}}_{n}$ for $n=0,1, \ldots, 6$, with the $\mathrm{SU}(2) \times \mathrm{SO}(3)$ representations of the vacuum. It is easy to see that $\overline{\mathrm{B}}$ contains particles with all spins and isospins in the range $\left|s-\frac{3}{2}\right| \leqslant s^{\prime} \leqslant\left|s+\frac{3}{2}\right|$ and $|J-2| \leqslant J^{\prime} \leqslant|J+2|$.

If further restrictions are placed on the vacuum quantum numbers, then the norms of the vectors in $\mathrm{V}$ which correspond to certain $\mathrm{SO}(3,2) \times \mathrm{SO}(3)$ representations vanish. This means that the $\operatorname{Osp}(3,4)$ representation does not contain these components. This is the phenomenon of multiplet shortening.

In the following sections, we describe the calculation of norms in the various $\overline{\mathbf{B}}_{n}$ sectors, and the reader will see the structure described above emerge. Actually, a complete and systematic calculation of norms in the higher $\overline{\mathrm{B}}_{n}$ sectors would be very tedious and exhausting even for the case $N=3$ we are considering; a similar systematic investigation for $N=8$ along the same lines does not appear possible without more sophisticated methods and presumably requires the help of a computer. Fortunately, for $N=3$, we can establish the existence of short multiplets by supplementing some norm calculations with rigorous but indirect arguments of the type mentioned in the introduction, using the decomposition into known $O \operatorname{sp}(1,4)$ multiplets and the Wigner-Inönü contraction. Similar arguments will also be of help for $N=8$, since they imply rather strong restrictions as will be discussed in the last section of this paper.

\section{The $\bar{B}_{1}$ sector}

We use standard Condon-Shortley phase conventions throughout the calculations with a non-conventional definition of reduced matrix element, to wit

$$
\left\langle B, j^{\prime}, m^{\prime}\left|T_{\mu}^{k}\right| A^{j m}\right\rangle=\left(B, j^{\prime}\left|T^{k}\right| A, j\right)\left\langle j, m, k, \mu \mid j^{\prime}, m^{\prime}\right\rangle,
$$

where the last factor is a standard Clebsch-Gordan coefficient.

The action of the highest-weight operator $\bar{a}_{1}^{+}$on the vacuum multiplet can be represented by

$$
\begin{aligned}
\bar{a}_{1}^{+}|\mathrm{vac}, m, M\rangle= & \sum_{\mu, \nu}=\left|\left(E_{0}+\frac{1}{2}, s+\mu, J+\nu\right), E_{0}+\frac{1}{2}, s+\mu, m+\frac{1}{2}, M+1\right\rangle \\
& \times R_{\mu \nu}\left\langle s, m, \frac{1}{2}, \frac{1}{2} \mid s+\mu, m+\frac{1}{2}\right\rangle\langle J, M, 1,1 \mid J+\nu, M+1\rangle,
\end{aligned}
$$

where the sum extends over the six possible values of quantum numbers $s^{\prime}$ and $J^{\prime}$ 
which can appear on the right side, i.e. $\mu= \pm \frac{1}{2}$ and $\nu=1,0,-1$. The $R_{\mu \nu}$ are reduced matrix elements. The Clebsch-Gordan coefficients which we use in (18) are tabulated in ref. [13]. To calculate the reduced matrix elements in (18), we begin by putting $m=s$ and $M=J$ so that only the highest state with $s^{\prime}=s+\frac{1}{2}$ and $J^{\prime}=J+1$ contributes. We then calculate the norm, using (11),

$$
\begin{aligned}
\left|R_{\frac{1}{2}, 1}\right|^{2} & =\left\langle\operatorname{vac}, s, J\left|\left(\bar{a}_{1}^{+}\right)^{*} \bar{a}_{1}^{+}\right| \text {vac, } s, J\right\rangle \\
& =\left\langle\operatorname{vac}, s, J\left|\left(M_{04}+J_{3}-T^{3}\right)\right| \operatorname{vac}, s, J\right\rangle \\
& =E_{0}+s-J .
\end{aligned}
$$

By taking, in turn, the values $m=-s, M=J ; m=s, M=J-1 ; m=-s, M=J-$ $1 ; m=s, M=-J$; and $m=-s, M=-J$, one finds the reduced matrix elements $R_{-\frac{1}{2}, 1} ; R_{\frac{1}{2}, 0} ; R_{-\frac{1}{2}, 0} ; R_{+\frac{1}{2},-1}$; and $R_{-\frac{1}{2},-1}$ in a tedious but straightforward calculation. The final result for the reduced matrix elements reads

$$
\begin{gathered}
\left|R_{\frac{1}{2}, 1}\right|^{2}=E_{0}+s-J, \\
\left|R_{-\frac{1}{2}, 1}\right|^{2}=E_{0}-s-J-1, \\
\left|R_{\frac{1}{2}, 0}\right|^{2}=E_{0}+s+1, \\
\left|R_{-\frac{1}{2}, 0}\right|^{2}=E_{0}-s, \\
\left|R_{\frac{1}{2},-1}\right|^{2}=E_{0}+s+J+1, \\
\left|R_{-\frac{1}{2},-1}\right|^{2}=E_{0}-s+J .
\end{gathered}
$$

The condition for positive norms is (for $s \neq 0$ )

$$
E_{0}>s+J+1 \text {, }
$$

and, if this condition is satisfied, all of the $\mathrm{SO}(3,2) \times \mathrm{SO}(3)$ representations in (18) are present. When higher $\overline{\mathrm{B}}_{n}$ sectors are considered, this condition leads to long representations.

One also finds in (20) several possibilities to eliminate states. For example, we can take $J=0$ and $E_{0}=s+1$. Then, because $R_{-\frac{1}{2}, 1}=0$ and only isospin 1 can occur, the only $\mathrm{SO}(3,2) \times \mathrm{SO}(3)$ representation contained in the sector $\bar{a}_{\alpha}^{i}|\mathrm{vac}, m, M\rangle$ is $D\left(s+\frac{3}{2}, s+\frac{1}{2}, 1\right)$. Although we do not follow this possibility through higher sectors, it seems clear that it leads to the massless $\operatorname{Osp}(3,4)$ representations discussed in [12]. 
We are primarily interested in massive short representations, and the simplest possibility is to take

$$
s=0, \quad E_{0}=J
$$

In this case, only spin $\frac{1}{2}$ occurs and $R_{\frac{1}{2}, 1}=0$. Thus one sees that the only $\operatorname{SO}(3,2) \times$ $\mathrm{SO}(3)$ representations contained in the $\overline{\mathrm{B}}_{1}$ sector are $D\left(J+\frac{1}{2}, \frac{1}{2}, J\right)$ and $D\left(J+\frac{1}{2}, \frac{1}{2}, J-1\right)$. Specifically the amplitude for finding other lowest-weight states among

$$
\bar{a}_{\alpha}^{-i}|\operatorname{vac}, 0, M\rangle,
$$

vanishes, which means that such representations are absent.

\section{The $\overline{\mathbf{B}}_{\mathbf{2}}$ sector}

The 15 operators in this sector can be classified according to their spin and isospin content as follows,

$$
\begin{gathered}
\varepsilon_{\alpha \beta}\left(\bar{a}_{\alpha}^{\left(i \bar{a}_{\beta}\right)}-\frac{1}{3} \delta^{i j} \bar{a}_{\alpha}^{k} \bar{a}_{\beta}^{k}\right), \\
\Delta J=2, \quad \Delta s=0, \quad 5 \text { operators, } \\
\varepsilon^{i j k} \bar{a}_{(\alpha}^{j} \bar{a}_{\beta)}^{k}, \\
\Delta J=1, \quad \Delta s=1, \quad 9 \text { operators, } \\
\varepsilon_{\alpha \beta} \bar{a}_{\alpha}^{i} \bar{a}_{\beta}^{i}, \\
\Delta J=\Delta s=0, \quad 1 \text { operator } .
\end{gathered}
$$

For later convenience, we define

$$
\begin{aligned}
\bar{a}_{\alpha}^{ \pm} & =\mp \sqrt{\frac{1}{2}}\left(\bar{a}_{\alpha}^{1} \pm i \bar{a}_{\alpha}^{2}\right), \\
a_{\alpha}^{ \pm} & =\mp \sqrt{\frac{1}{2}}\left(a_{\alpha}^{1} \pm i a_{\alpha}^{2}\right), \\
\left(\bar{a}_{\alpha}^{ \pm}\right)^{*} & =-a_{\alpha}^{\mp} .
\end{aligned}
$$

We will also use $J^{ \pm}=J_{1} \pm i J_{2}, T^{ \pm}=T^{1} \pm i T^{2}$. We study first the states obtained by applying the $\Delta T^{3}=2$ component of (22a), namely $\varepsilon_{\alpha \beta} \bar{a}_{\alpha}^{+} \bar{a}_{\beta}^{+}$, to the vacuum multi- 
plet. Such states can be expressed as

$\varepsilon_{\alpha \beta} \bar{a}_{\alpha}^{+} \bar{a}_{\beta}^{+}|\operatorname{vac}, m, M\rangle$

$$
\begin{aligned}
& =\left|\left(E_{0}+1, s, J+2\right), E_{0}+1, s, m, M+2\right\rangle R_{2}\langle J, M, 2,2 \mid J+2, M+2\rangle \\
& +\left|\left(E_{0}+1, s, J+1\right), E_{0}+1, s, m, M+2\right\rangle R_{1}\langle J, M, 2,2 \mid J+1, M+2\rangle \\
& + \text { states of isospin } J, J-1, J-2 .
\end{aligned}
$$

After tedious but straightforward operator manipulations we find the result

$$
\begin{aligned}
& \left\langle\operatorname{vac}, m^{\prime}, M^{\prime}\left|\left(\varepsilon_{\gamma \delta} \bar{a}_{\gamma}^{+} \bar{a}_{\delta}^{+}\right)^{*}\left(\varepsilon_{\alpha \beta} \bar{a}_{\alpha}^{+} \bar{a}_{\beta}^{+}\right)\right| \operatorname{vac}, m, M\right\rangle \\
& \quad=4\left\langle\operatorname{vac}, m^{\prime}, M^{\prime}\left|\left\{\left(M_{04}-T^{3}\right)^{2}-\left(M_{04}-T^{3}\right)-J_{k} J_{k}\right\}\right| \operatorname{vac}, m, M\right\rangle .
\end{aligned}
$$

We note that the norms of all $2 J+1$ vectors on the left side of (24) are positive if the condition $E_{0}>s+J+1$ is satisfied. This is the same as the condition encountered in the $\overline{\mathrm{B}}_{1}$ sector which we identified as the condition for a massive long representation if $s>0$. We now assume the conditions $s=0$ and $E_{0}=J$ to explore further the structure of short representations. For the value $M=J$ only the isospin $J+2$ state contributes in (24) and we find from (25) that $R_{2}=0$. We then take $M=J-1$ for which only the isospin $J+1$ state can contribute; then we find that $R_{1}=0$. This means that the $\mathrm{SO}(3,2) \times \mathrm{SO}(3)$ representations $D\left(E_{0}+1,0, J+2\right)$ and $D\left(E_{0}+1,0, J+1\right)$ do not occur in the short $\operatorname{Osp}(3,4)$ multiplets we are constructing. We postpone until later the study of lower isospin states in (24).

To simplify further calculations we assume the conditions $s=0$ and $E_{0}=J$ of short representations from now on and denote the vacuum states simply by $|v a c, M\rangle$. We can now explore states obtained by applying operators of (22b) to the vacuum multiplet. Since these states all have spin 1 , we call this the vector sector.

For the highest-weight operator, we actually have $\left[\bar{a}_{1}^{+}, \bar{a}_{1}^{3}\right]=2 \bar{a}_{1}^{+} \bar{a}_{1}^{3}$ since the anticommutator vanishes according to (11). Therefore we consider the states $\bar{a}_{1}^{+} \bar{a}_{1}^{3} \mid$ vac, $\left.M\right\rangle$ which can be expressed as

$$
\begin{aligned}
\bar{a}_{1}^{+} \bar{a}_{1}^{3}|\operatorname{vac}, M\rangle= & \sum_{\nu}|(J+1,1, J+\nu) J+1,1,1, M+1\rangle R_{\nu}\langle J, M, 1,1 \mid J+\nu, M+1\rangle \\
& +\bar{a}_{1}^{i} \bar{a}_{1}^{i}|\operatorname{vac}, M+1\rangle C\langle J, M, 1,1 \mid J, M+1\rangle .
\end{aligned}
$$

The $R_{v}$ are reduced matrix elements and the sum extends over the values $\nu=1,0,-1$ for the possible lowest-weight states of the $\mathrm{SO}(3,2) \times \mathrm{SO}(3)$ representations which can occur. The last term in (26) is a possible admixture of the state $M_{+}^{+} \mid$vac, $\left.M+1\right\rangle$ which, of course, has total isospin $J$. The reason such admixtures occur is that an 
operator of $\overline{\mathbf{B}}_{n}$ can create a superposition of all states of the appropriate quantum numbers from the vacuum including excited states of $\mathrm{SO}(3,2) \times \mathrm{SO}(3)$ multiplets with $E_{0}<J+\frac{1}{2} n$. In the $\overline{\mathrm{B}}_{2}$ sector such states necessarily have isospin $J$, which is why these admixtures did not occur in the calculation of $J+2$ and $J+1$ components of (24). Note that the boost admixture is orthogonal to the lowest-weight states in (26) because it belongs to a different representation of $\mathrm{SO}(3,2) \times \mathrm{SO}(3)$.

The action of $\operatorname{SO}(3,2)$ energy deboosts

$$
M_{k}^{-}=-\left(M_{k}^{+}\right)^{*}=\left(i M_{0 k}+M_{k 4}\right)=\frac{1}{3}\left(\varepsilon \sigma_{k}\right)_{\alpha \beta} a_{\alpha}^{i} a_{\beta}^{i},
$$

can be used to determine the coefficient $\sigma$. Clearly $M_{k}^{-}$annihilates the summation term in (26) which contains lowest-weight states. We calculate the effect of $M_{k}^{-}$on the remaining terms in (26) using

$$
\left[M_{k}^{-}, \bar{a}_{\alpha}^{i}\right]=\left(\varepsilon \sigma_{k}\right)_{\alpha \beta} a_{\beta}^{i},
$$

which follows from (2). On the left side of (26) we find

$$
\begin{aligned}
\left.M_{k}^{-} \bar{a}_{1}^{+} a_{1}^{3} \mid \text { vac, } M\right\rangle & \left.\left.=\left(\varepsilon \sigma_{k}\right)_{1 \beta}\left\{a_{\beta}^{+}, \bar{a}_{1}^{3}\right\} \mid \text { vac, } M\right\rangle=\sqrt{\frac{1}{2}}\left(\varepsilon \sigma_{k}\right)_{11} T^{+} \mid \text {vac, } J-1\right\rangle \\
& =\sqrt{\frac{1}{2}}\left(\varepsilon \sigma_{k}\right)_{11} \sqrt{(J+M+1)(J-M)}|\operatorname{vac}, M+1\rangle .
\end{aligned}
$$

From the right side of (26) we obtain

$$
\begin{aligned}
M_{k}^{-} \bar{a}_{1}^{i} \bar{a}_{1}^{i}|\mathrm{vac}, M+1\rangle C\langle J, M, 1,1 \mid J, M+1\rangle \\
\quad=\left(\varepsilon \sigma_{k}\right)_{1 \beta}\left\{a_{\beta}^{i}, \bar{a}_{1}^{i}\right\}|\mathrm{vac}, M+1\rangle C\langle J, M, 1,1 \mid J, M+1\rangle \\
\quad=\left(\varepsilon \sigma_{k}\right)_{11} 3 M_{04}|\mathrm{vac}, M+1\rangle C\langle J, M, 1,1 \mid J, M+1\rangle .
\end{aligned}
$$

Since (29) and (30) must be equal, we find, after inserting the value of $\langle J, M, 1,1 \mid J, M+1\rangle$, that $C=-\sqrt{(J+1) / 9 J}$.

We must now calculate the norms of the states in (26). A tedious but straightforward operator calculation gives

$$
\begin{aligned}
& \left\langle\operatorname{vac}, M^{\prime}\left|\left(\bar{a}_{1}^{+} \bar{a}_{1}^{3}\right)^{*}\left(\bar{a}_{1}^{+} \bar{a}_{1}^{3}\right)\right| \operatorname{vac}, M\right\rangle \\
& =\left\langle\operatorname{vac}, M^{\prime}\left|\left\{\left(M_{04}+J_{3}\right)\left(M_{04}+J_{3}-T^{3}+1\right)-\frac{1}{2} T^{+} T^{-}\right\}\right| \operatorname{vac}, M\right\rangle .
\end{aligned}
$$

For the value $M=J$ only the isospin $J+1$ state in (26) contributes, and one finds 
$R_{1}=0$, indicating that there are no spin-1 particles with isospin $J+1$ in the short representations. For the value $M=J+1$ the norm calculation gives

$$
1=\left(R_{0}^{2}+C^{2}\left\langle\operatorname{vac}, J\left|a_{1}^{i} a_{1}^{i} \bar{a}_{1}^{j} \bar{a}_{1}^{j}\right| \operatorname{vac}, J\right\rangle\right)\langle J, J-1,1,1 \mid J, J\rangle^{2} .
$$

The expectation value in (32) can be calculated by tedious but straightforward methods, which give the result

$$
\left\langle\operatorname{vac}, M^{\prime}\left|a_{1}^{i} a_{1}^{i} \bar{a}_{1}^{j} \bar{a}_{1}^{j}\right| \mathrm{vac}, M\right\rangle=9\left\langle\operatorname{vac}, M^{\prime}\left|\left(M_{04}+J_{3}\right)\right| \operatorname{vac}, M\right\rangle=9 J \delta_{M^{\prime} M} \text {. }
$$

When this is inserted in (32) together with the values of $C$ and $\langle J, J-1,1,1 \mid J, J\rangle$, one finds that $R_{0}=0$, indicating that there is no isospin $J$ spin-1 particle in the $\overline{\mathrm{B}}_{2}$ sector.

Finally, we take the $M=J-2$ and calculate the norm of (26). We obtain, using (31),

$$
\begin{aligned}
3= & R_{-1}^{2}\langle J, J-2,1,1 \mid J-1, J-1\rangle^{2} \\
& +C^{2}\left\langle\mathrm{vac}, J-1\left|a_{1}^{i} a_{1}^{i} \bar{a}_{1}^{i} \bar{a}_{1}^{j}\right| \mathrm{vac}, J-1\right\rangle\langle J, J-2,1,1 \mid J, J-1\rangle^{2} .
\end{aligned}
$$

Inserting values, we find that the second term in (34) is smaller than 2 for all $J \geqslant 1$. Then $R_{-1}^{2}$ is positive, so that the short representations contain a massive vector particle in the $\operatorname{SO}(3,2) \times \operatorname{SO}(3)$ representations $(J+1,1, J-1)$. This completes the analysis of the vector sector.

We now return to the question of lower isospin spin-0 particles in the sector (22a). It is best to consider at the same time the sector (22c), since both sectors can contain particles in the $\mathrm{SO}(3,2) \times \mathrm{SO}(3)$ representation $(J+1,0, J)$. Let us designate by $E^{(\mu)}$, the five $\Delta T^{3}=\mu$ components of (22a) with $E^{(2)}=\varepsilon_{\alpha \beta} \bar{a}_{\alpha}^{+} \bar{a}_{\beta}^{+}$and the rest obtained by repeated lowering with $T^{-}$, and let $F=\varepsilon_{\alpha \beta} \bar{a}_{\alpha}^{i} \bar{a}_{\beta}^{i}$ denote the operator of (22c).

There is the possibility that two independent $(J+1,0, J)$ representations occur. If the vectors

$$
|\Psi, M\rangle=\sum_{\mu}\langle J, M-\mu, 2, \mu \mid J, \mu\rangle E^{(\mu)}|\mathrm{vac}, M-\mu\rangle
$$

and $F \mid$ vac, $M\rangle$ are linearly independent, then one can find coefficients $A, B, C, D$ and form two orthogonal lowest-weight states

$$
\begin{aligned}
& |(J+1,0, J) J+1,0,0, M\rangle=A|\Psi, M\rangle+B F|\operatorname{vac}, M\rangle \\
& |(J+1,0, J) J+1,0,0, M\rangle^{\prime}=C|\Psi, M\rangle+D F|\operatorname{vac}, M\rangle
\end{aligned}
$$


Two copies of the representation $(J+1,0, J)$ will then be present in the $\operatorname{Osp}(3,4)$ short multiplets. Note that there are no boost admixtures in (35) because there are no states of the appropriate quantum numbers $\omega=E_{0}+1, j^{\prime}=0$ in the weight diagram of the scalar representation $\left(E_{0}, 0\right)$ of $\mathrm{SO}(3,2)$.

Short representations will be all the shorter if the doubling of $(J+1,0, J)$ components does not occur, and we now show that this is the case, since the vectors $|\Psi, M\rangle$ and $F \mid$ vac, $M\rangle$ are linearly dependent. To prove this, we first note that, as a consequence of the absence of $J+2$ and $J+1$ states in (24), and the inverse Clebsch-Gordan expansion, the three vectors

$$
\frac{1}{\langle J, J-\mu, 2, \mu \mid J, J\rangle} E^{(\mu)}|\operatorname{vac}, J-2\rangle,
$$

for $\mu=2,1,0$ are actually equal. If we use this and the orthogonality relations of Clebsch-Gordan coefficients, we find that

$$
|\Psi, J\rangle=\frac{1}{\langle J, J-2,2,2 \mid J J\rangle} E^{(2)}|\mathrm{vac}, J-2\rangle .
$$

Hence if we can show that $E^{(2)}|\mathrm{vac}, J-2\rangle$ and $F|\mathrm{vac}, J\rangle$ are linearly dependent, the desired dependence of $|\Psi, M\rangle$ and $F|\mathrm{vac}, M\rangle$ then follows by lowering with $T^{-}$.

The vectors $E^{(2)} \mid$ vac, $\left.J-2\right\rangle$ and $F|\operatorname{vac}, J\rangle$ are linearly dependent if there is a complex constant $A$ such that the superposition

$$
|\Phi\rangle=\varepsilon_{\alpha \beta} \bar{a}_{\alpha}^{i} \bar{a}_{\beta}^{i}|\mathrm{vac}, J\rangle+A \varepsilon_{\alpha \beta} \bar{a}_{\alpha}^{+} \bar{a}_{\beta}^{+}|\mathrm{vac}, J-2\rangle,
$$

has zero norm. The norm may be calculated using (25) and the analogous relations

$$
\begin{aligned}
& \left\langle\operatorname{vac}, M^{\prime}\left|\left(\varepsilon_{\alpha \beta} \bar{a}_{\alpha}^{i} \bar{a}_{\beta}^{i}\right)^{*} \varepsilon_{\gamma \delta} \bar{a}_{\gamma}^{j} \bar{a}_{\delta}^{j}\right| \operatorname{vac}, M\right\rangle \\
& =\left\langle\operatorname{vac}, M^{\prime}\left|6 M_{04}\left(2 M_{04}+1\right)-8 T^{i} T^{i}-12 J_{k} J_{k}\right| \operatorname{vac}, M\right\rangle .
\end{aligned}
$$$$
\left\langle\operatorname{vac}, M^{\prime}\left|\varepsilon_{\alpha \beta} a_{\alpha}^{i} a_{\beta}^{i} \varepsilon_{\gamma \delta} \bar{a}_{\gamma}^{+} \bar{a}_{\delta}^{+}\right| \operatorname{vac}, M\right\rangle=-\left\langle\operatorname{vac}, M^{\prime}\left|2 T^{+} T^{+}\right| \mathrm{vac}, M\right\rangle \text {. }
$$

One then finds

$$
\langle\Phi \mid \Phi\rangle=8\left\{\left(\operatorname{Re} A+\frac{1}{2} \sqrt{J(2 J-1)}\right)^{2}+(\operatorname{Im} A)^{2}\right\},
$$

which vanishes for the real value $A=-\frac{1}{2} \sqrt{J(2 J-1)}$. Thus our vectors are linearly dependent, and there is one and only one $(J+1,0, J)$ particle in the $\mathrm{B}_{2}$ sector. 
Finally, we can complete the study of lower isospin states in (24), and we can now write (24) as

$$
\begin{aligned}
\varepsilon_{\alpha \beta} \bar{a}_{\alpha}^{+} & \bar{a}_{\beta}^{+}|\operatorname{vac}, M\rangle \\
& =\sum_{\mu}\left|\left(E_{0}+1, s, J+\mu\right) E_{0}+1,0,0, M+2\right\rangle R_{\mu}\langle J, M, 2,2 \mid J+\mu, M+2\rangle .
\end{aligned}
$$

We already know that $R_{2}=R_{1}=0$ and that $R_{0} \neq 0$. To investigate the lower isospin components we simply take $M=J-2, J-3$ and $J-4$ in turn, and use (25) and explicit values of the isospin 2 Clebsch-Gordan coefficients [14] to calculate $R_{0}, R_{-1}$ and $R_{-2}$. We obtain positive values for the squares of these reduced matrix elements (for $J \geqslant 2$ where the calculation is valid), which indicates that the representations $(J+1,0, J) ;(J+1,0, J-1)$ and $(J+1,0, J-2)$ are present in the short $\operatorname{Osp}(3,4)$ multiplets with positive norm. This completes the analysis of the $\overline{\mathrm{B}}_{2}$ sector.

\section{The $\overline{\mathbf{B}}_{3}$ sector: absence of $\operatorname{spin} \frac{3}{2}$}

The $\overline{\mathrm{B}}_{3}$ sector contains 20 operators which can be classified in three distinct representations of $\mathrm{SU}(2) \times \mathrm{SO}(3)$ (where the first factor refers to spatial rotations and the second to isospin), namely $\Delta s=\frac{3}{2}, \Delta J=0$; and $\Delta s=\frac{1}{2}, \Delta J=2$; and $\Delta s=\frac{1}{2}$, $\Delta J=1$. It would be very tedious to study all of these operators, and fortunately we will not have to. However, it is important to show that the short representations do not contain spin- $\frac{3}{2}$ particles and therefore we examine the $\Delta s=\frac{3}{2}, \Delta J=0$ multiplet which contains the only operators which can lead to $s=\frac{3}{2}$ states.

The highest-weight operator of this multiplet is

$$
\varepsilon^{i j k} \bar{a}_{1}^{i} \bar{a}_{1}^{j} \bar{a}_{1}^{k}
$$

The action of this operator on the vacuum multiplet may be expressed as

$$
\begin{aligned}
& \varepsilon^{i j k} \bar{a}_{1}^{i} \bar{a}_{1}^{j} \bar{a}_{1}^{k}|\operatorname{vac}, M\rangle \\
& \quad=R\left|\left(J+\frac{3}{2}, \frac{3}{2}, J\right) J+\frac{3}{2}, \frac{3}{2}, \frac{3}{2}, M\right\rangle+S\left(\bar{a}_{1}^{i} \bar{a}_{1}^{i}\right)\left|\left(J+\frac{1}{2}, \frac{1}{2}, J\right) J+\frac{1}{2}, \frac{1}{2}, M\right\rangle,
\end{aligned}
$$

where we have the possibility of a superposition of the lowest-weight state of a new spin $\frac{3}{2}$ multiplet and an orthogonal admixture of the known $\left(J+\frac{1}{2}, \frac{1}{2}, J\right)$ particle with the appropriate $\Delta J_{3}=+1$ component of the energy boost operator applied. The energy deboosts $M_{k}^{-}$of (29) annihilate the first term on the right side of (43) and, just as in the previous analysis of the vector sector, they may now be used to determine the coefficient $S$. 
We apply $M_{k}^{-}$to the left side of (43) and use (28) and some more manipulations of $a_{k}^{i}$ operators past $\bar{a}_{1}^{j}$ operators, to obtain

$$
M_{k}^{-} \varepsilon^{i j k} \bar{a}_{1}^{i} \bar{a}_{1}^{j} a_{1}^{k}|\mathrm{vac}, M\rangle=6 i\left(\varepsilon \sigma_{k}\right)_{11} \bar{a}_{1}^{m} T^{m}|\mathrm{vac}, M\rangle .
$$

By a similar calculation we obtain from the right side of (43) the result

$$
\begin{aligned}
& M_{k}^{-} S \bar{a}_{1}^{i} \bar{a}_{1}^{i}\left|\left(J+\frac{1}{2}, \frac{1}{2}, J\right), J+\frac{1}{2}, \frac{1}{2}, \frac{1}{2}, M\right\rangle \\
& \quad=3 i S\left(\varepsilon \sigma_{k}\right)_{11}(J+1)\left|\left(J+\frac{1}{2}, \frac{1}{2}, J\right) J+\frac{1}{2}, \frac{1}{2}, \frac{1}{2}, M\right\rangle .
\end{aligned}
$$

By using the reduced matrix element $R_{\frac{1}{2}, 0}$ of (20) and the isospin Clebsch-Gordan coefficients as in (18) one finds that

$$
\bar{a}_{1}^{m} T^{m}|\operatorname{vac}, \bar{M}\rangle=\sqrt{J}(J+1)\left|\left(J+\frac{1}{2}, \frac{1}{2}, J\right) J+\frac{1}{2}, \frac{1}{2}, \frac{1}{2}, M\right\rangle,
$$

then (44) and (45) can be compared to give the value $s=2 \sqrt{J}$ (times an arbitrary phase factor which can be ignored).

We must now calculate the norm of the vectors in (43). By a long but straightforward operator calculation, one calculates that the norm of the left side in

$$
\left.\| \varepsilon_{i j k} \bar{a}_{1}^{i} a_{1}^{j} \bar{a}_{1}^{k} \mid \text { vac, } M\right\rangle \|^{2}=36 J(J+1) .
$$

The norm of the right side can be calculated by slightly less tedious means to give the result

$$
|| \text { r.h.s. of }(43)||^{2}=|R|^{2}+9(J+1)|S|^{2} .
$$

Inserting the value of $S$, we find that $R=0$ indicating that there are no spin- $\frac{3}{2}$ particles in the $B_{3}$ sector. However, it is easy to see that the 6 operators of the $B_{5}$ sector have $\Delta s=\frac{1}{2}$ and cannot create spin- $\frac{3}{2}$ particles. Hence we may actually now conclude that the short representations of $\operatorname{Osp}(3,4)$ indeed have maximum spin 1 .

\section{Osp $(1,4)$ decomposition}

In sects. $4-6$, it has been shown that the short $O s p(3,4)$ representations under study contain the following $\mathrm{SO}(3,2) \times \mathrm{SO}(3)$ components:

$$
\begin{array}{lll}
\overline{\mathrm{B}}_{0} \text { sector } & (J, 0, J), \\
\overline{\mathrm{B}}_{1} \text { sector } & \left(J+\frac{1}{2}, \frac{1}{2}, J\right), \quad\left(J+\frac{1}{2}, \frac{1}{2}, J-1\right), \\
\overline{\mathrm{B}}_{2} \text { sector } & (J+1,0, J), \quad(J+1,0, J-1), \quad(J+1,0, J-2), \\
& (J+1,1, J-1) .
\end{array}
$$


The $\overline{\mathbf{B}}_{n}$ sectors for $n \geqslant 3$ may contain additional particles. On the other hand, Osp $(3,4)$ certainly contains $\operatorname{Osp}(1,4)$ as a subalgebra and the representations we are interested can certainly be decomposed into massive type A and type B representations of $\operatorname{Osp}(1,4)$, discussed in sect. 2 , each with definite isospin. It is clear that the unique way to group the particles above in $\mathrm{Osp}(1,4)$ representations is as follows:

$$
\begin{gathered}
(J) \times\left\{D(J, 0) \oplus D\left(J+\frac{1}{2}, \frac{1}{2}\right) \oplus D(J+1,0)\right\}, \\
(J-1) \times\left\{D\left(J+\frac{1}{2}, \frac{1}{2}\right) \oplus D(J+1,1) \oplus D(J+1,0) \oplus D\left(J+\frac{3}{2}, \frac{1}{2}\right\},\right. \\
(J-2) \times\left\{D(J+1,0) \oplus D\left(J+\frac{3}{2}, \frac{1}{2}\right) \oplus D(J+2,0)\right\} .
\end{gathered}
$$

Note that we have added two spin- $\frac{1}{2}$ particles with $E_{0}=J+\frac{3}{2}$ and one spin-0 particle with $E_{0}=J+2$ to those of the previous list in order to complete the $\operatorname{OSp}(1,4)$ multiplets. We can be confident that the corresponding lowest-weight states are contained, with positive norm, in the $\bar{B}_{3}$ and $\bar{B}_{4}$ sectors of the Hilbert space $\mathrm{V}$.

We do not know yet whether the higher $\overline{\mathrm{B}}_{n}$ sectors contain additional particle states, but it is clear that such states would form new $\operatorname{OSp}(1,4)$ massive multiplets which begin with $E_{0}=J+\frac{3}{2}$ or $E_{0}=J+2$, i.e. in the $\overline{\mathrm{B}}_{3}$ or $\overline{\mathrm{B}}_{4}$ sectors. The possibilities are actually fairly limited. For example, a multiplet beginning at the $\overline{\mathbf{B}}_{4}$ level would include a particle at the $\bar{B}_{6}$ level which necessarily has the quantum numbers $(J+3,0, J)$, and there can only be one such particle since there is only one operator in $\overline{\mathrm{B}}_{6}$ (which has the quantum numbers $\Delta J=\Delta s=0$ ). Thus we have the possibility of $(2 J+1)$ massive Wess-Zumino representations of $\operatorname{OSp}(1,4)$, with the particle content

$$
(J) \times\left\{D(J+2,0) \oplus D\left(J+\frac{5}{2}, \frac{1}{2}\right) \oplus D(J+3,0)\right\} .
$$

Each multiplet beginning at the $B_{3}$ level would include a particle at the $B_{5}$ level with possible quantum numbers $\left(J+\frac{3}{2}, \frac{1}{2}, I\right)$ with isospin $I=J+1, J$, or $J-1$. Such particles could be obtained from the set of six operators of $\overline{\mathrm{B}}_{5}$, with $\Delta J=1$ and $\Delta s=\frac{1}{2}$, applied to the vacuum. Thus we have the possibility of type B, $s=\frac{1}{2}$ multiplets of $\operatorname{OSp}(1,4)$ with particle content

$$
\begin{array}{r}
(J+1) \times\left\{D\left(J+\frac{3}{2}, \frac{1}{2}\right) \oplus D(J+2,1) \oplus D(J+2,0) \oplus D\left(J+\frac{5}{2}, \frac{1}{2}\right)\right\}, \\
(J-1) \times\{\text { idem }\}, \quad(J) \times\{\text { idem }\} .
\end{array}
$$

Further, since there is at most one particle with quantum numbers $\left(J+\frac{5}{2}, \frac{1}{2}, J\right)$ in the $\overline{\mathbf{B}}_{5}$ sector the Wess-Zumino multiplets of (51) and the isospin $(J)$ multiplet in (52) cannot be simultaneously present.

At this point the prospects seem dismal. It would take still more tedious (but straightforward) calculations in the higher $\overline{\mathrm{B}}_{n}$ sectors to establish whether the short $\mathrm{OSp}(3,4)$ representations contain some of the components in $(51),(52)$. Fortunately 
these calculations can be avoided. There is a simple indirect argument which shows that none of the components in (51), (52) are present, as we shall see in sect. 9.

\section{The Poincaré limit and the general Higgs procedure}

We now use the fact that in the limit that the radius of anti de Sitter space tends to infinity any irreducible representation of $\operatorname{OSp}(3,4)$ must describe a massless representation, possibly reducible, of $N=3$ Poincaré supersymmetry. Since we know that the short $\operatorname{OSp}(3,4)$ representations contain no spin- $\frac{3}{2}$ particles, this limit must involve only $s_{\max }=1$ Poincaré representations. Such representations are well-known [5] to have the structure of a Fock vacuum of helicity 1 and arbitrary isospin I and helicity states, three $\lambda=\frac{1}{2}$, three $\lambda=0$ and one $\lambda=-\frac{1}{2}$ obtained by applying anti-symmetric contributions of three creation operators. After the usual CPT doubling one sees that this representation contains the following particles

$$
\begin{array}{ll}
\operatorname{spin} 1 & I \times 0=I, \\
\operatorname{spin} \frac{1}{2} & I \times(0 \oplus 1)=I+1 \oplus I \oplus I-1 \oplus I, \\
\operatorname{spin} 0 & I \times(1 \oplus 1)=I+1 \oplus I \oplus I-1 \oplus I+1 \oplus I \oplus I-1 .
\end{array}
$$

To compare this list of particles with those of a massive $O \operatorname{sp}(3,4)$ representation we must combine an $I$ of spin- 0 states with the $I$ of spin- 1 states to account for all the states in the spin-1 weight diagram.

We now compare the list (53) with the list (50) of particles obtained from the low $\overline{\mathrm{B}}_{n}$ sectors of short $\operatorname{Osp}(3,4)$ representations together with the particles necessary to give complete $\operatorname{Osp}(1,4)$ multiplets. Clearly we must take $I=J-1$ so that the spin-1 states agree. We then see that the remaining nine particles in (50) and (53) agree perfectly in spin and isospin.

Since the particles in (50) already limit properly to an irreducible $s_{\max }=1$ massless representation of $N=3$ Poincare supersymmetry, any additional particles in (51) and (52) must separately limit to a similar representation. However, it is easy to see there are simply too few possible spin-0 particles in (51), (52) to obtain a combination of the representations (48) in the limit. This means that none of the possible particles in (51), (52) are actually present in the short $\operatorname{OSp}(3,4)$ representations, and the $\mathrm{OSp}(1,4)$ decomposition in $(50)$ actually gives the complete particle content.

Since the argument based on the Poincare limit is somewhat indirect it is desirable to check the important conclusion that the possible multiplets of (51) and (52) are absent. To do this we note that all these $O \operatorname{sp}(1,4)$ representations contain $D\left(J+\frac{5}{2}, \frac{1}{2}\right)$ components whose lowest-weight states would be contained in the $\bar{B}_{5}$ sector of the vector space $\mathrm{V}$. We have therefore studied the operator $\bar{a}_{1}^{-} \bar{a}_{1}^{3} \bar{a}_{2}^{3} \bar{a}_{1}^{+} \bar{a}_{2}^{+}$applied to vacuum states. This operator is not totally antisymmetric, but it certainly can be expressed as a sum of a non-zero operator in $\overline{\mathbf{B}}_{5}$ plus sums of products of two energy 
boosts and single operators $\bar{a}_{\alpha}^{i}$. As in previous sections the action of this operator on the vacuum multiplet may be expressed as superpositions of possible lowest-weight states of representations $D\left(J+\frac{5}{2}, \frac{1}{2}, J+1\right), D\left(J+\frac{5}{2}, \frac{1}{2}, J\right)$ and $D\left(J+\frac{5}{2}, \frac{1}{2}, J-1\right)$ of $\mathrm{SO}(3,2) \times \mathrm{SO}(3)$ plus possible boost admixtures. After some norm calculations one can show that the isospin $J+1$ and $J$ states are absent. This limits the remaining $\operatorname{OSp}(1,4)$ multiplets in $(51)$ and $(52)$ to a single multiplet of isospin $J-1$ in $(52)$. We were unable, because of time constraints, to show that the last multiplet is absent, but the partial check of the indirect argument based on the Poincare limit is already non-trivial.

Thus we have obtained an infinite set of short massive representations of $\operatorname{Osp}(3,4)$ with $s_{\max }=1$. Each representation is built on a vacuum multiplet $D(J, 0, J)$ of $\mathrm{SO}(3,2) \times \mathrm{SO}(3)$, so that the energy is quantized in terms of the isospin. Note that this fact is in accord with expectations in a Kaluza-Klein theory. There one expects an infinite tower of massive states with masses determined by the eigenvalues of certain differential operators (e.g. the laplacian) on the internal space. Note also that isospin does not increase with increasing spin in the short representations. Instead, it tends to decrease, and the $s_{\max }=1$ particle occurs with unique isospin $J-1$.

Although we have used the term massive to describe the short $O s p(3,4)$ representations, it is strictly true that only for vacuum isospin values $J=\frac{3}{2}$, and $J \geqslant \frac{5}{2}$ that the representations contain only massive particles with mass interpreted in the sense of the group $\mathrm{SO}(3,2)$. For the value $J=2$ inspection of $(50)$ reveals the curious feature that the representation mixes massive and massless particles. This phenomenon is not new; it is already contained [6] in the type A representation of Osp $(1,4)$ with $E_{0}=2$. For $J=\frac{3}{2}$ one should note that the isospin $J-2$ particles in (50) are absent, thus giving a rather short representation involving massive particles. For $J=1$ the representation again appears to mix massive and massless particles; but here there is a subtlety since the type B representation in (50) is not correct for $J=1$. We believe that the norms of the $D(J+1,0, J-1)$ and $D\left(J+\frac{3}{2}, \frac{1}{2}, J-1\right)$ components of (50) vanish for $J=1$, and the representation is purely massless in this case. For $J=\frac{1}{2}$ only the isospin $J$ particles are present in (50) and we seem to have a representation which contains boson and fermion Dirac singletons. Although this discussion seems correct, it is desirable to study the $J=\frac{1}{2}, J=1$ and $J=\frac{3}{2}$ cases further since the determination of the scalar states in the $\overline{\mathbf{B}}_{2}$ sector was strictly valid only for $J \geqslant 2$.

The Poincaré limit has provided a very simple procedure to ascertain the spin and isospin structure of massive $\operatorname{Osp}(3,4)$ representations. We simply take a massless representation of $N=3$ Poincare supersymmetry, and use the Higgs mechanism to absorb some of the lower-spin states. With this in view we return to the fact that in the analysis of (16) we did not consider all the possibilities for eliminating states. For example, we could take a vacuum in the representation $D(s+J+1, s, J)$ which would eliminate one component of (20). This is again a condition which quantizes energy in terms of isospin (and spin). It seems reasonable to speculate that this leads 
to a representation of $\operatorname{Osp}(3,4)$ which limits to a single Poincaré representation with Fock vacuum of helicity $s+1$ and isospin $J$. Such a massive representation of Osp $(3,4)$ would then contain particles of spin $s+1, s+\frac{1}{2}, s, s-\frac{1}{2}$, which is still shorter than the expected spin range of long representations.

\section{Multiplet shortening in $\operatorname{Osp}(8,4)$}

Although the existence of short massive representations was established only for $N=3$ in the previous sections, our primary motivation was to understand the massive modes in 11-dimensional supergravity compactified on the round 7-sphere. The invariance group of this Kaluza-Klein background is $\operatorname{OSp}(8,4)$ [15]. Thus one needs to know that this superalgebra has massive short representations with $s_{\max }=2$. Although this can, in principle, be demonstrated using the techniques developed in the earlier sections for $N=3$, it is a far more tedious task. The results obtained for $N=3$ do make it extremely plausible that the desired representations do exist for $N=8$, and we will assume that this is the case. In the Poincare limit these representations must approach a massless representation of $N=8$ Poincaré supersymmetry with $s_{\max }=2$, and we will make the simplifying assumption that it is a single irreducible representation which is obtained. If the lower helicity Higgs states in this limit are suitably combined with maximum helicity, as is required for massive representations of $\mathrm{SO}(3,2)$, this limit then determines the spin and $\mathrm{SO}(8)$ structure of the desired short representations. This procedure was first used in [16] to determine the structure of the lowest massive level of compactified supergravity. In this section we will determine the structure of all the short $\operatorname{OSp}(8,4)$ representations which seem to be relevant.

The irreducible representations of $\mathrm{SO}(8)$ are uniquely characterized [17] by the Dynkin label $\left(a_{1}, a_{2}, a_{3}, a_{4}\right)$, where the $a_{i}$ are non-negative integers. The simplest massless representation of $N=8$ Poincare supersymmetry has the spin and $\operatorname{SO}(8)$ structure (for definiteness, we assign the spinor-charges to the $8_{\mathrm{s}}$ representation)

$$
\begin{array}{ll}
s=2, & 1=(0,0,0,0), \\
s=\frac{3}{2}, & 8_{\mathrm{s}}=(0,0,0,1), \\
s=1, & 28=(0,1,0,0), \\
s=\frac{1}{2}, & 56_{\mathrm{s}}=(1,0,1,0), \\
s=0, & 35_{\mathrm{v}}=(2,0,0,0), \\
& 35_{\mathrm{c}}=(0,0,2,0) .
\end{array}
$$

The general $s_{\max }=2$ massless representation is obtained by computing the direct product of the $\mathrm{SO}(8)$ representations above with an arbitrary representation $\left(a_{1}, a_{2}, a_{3}, a_{4}\right)$ describing an $s=2$ Fock vacuum. 
The Higgs procedure gives a strong constraint on the representations we are interested in, because we require that lower-helicity states for spin-2, spin- $\frac{3}{2}$, and spin-1 particles occur in exactly the same $S O(8)$ representations as the highest helicity states for these particles in order to combine states to get massive $\operatorname{SO}(3,2)$ representations for $s=2, s=\frac{3}{2}$, and $s=1$. Not all Fock vacuum representations satisfy these constraints. For example, if we take the Fock vacuum in the representation $8_{\mathrm{s}}$, then the massive spin-2 particle is in $8_{\mathrm{s}}$ but the spin-0 particles are in

$$
\begin{aligned}
& 8_{\mathrm{s}} \times 35_{\mathrm{v}}=56_{\mathrm{s}} \oplus 224_{\mathrm{vs}}, \\
& 8_{\mathrm{s}} \times 35_{\mathrm{c}}=56_{\mathrm{s}} \oplus 224_{\mathrm{cs}} .
\end{aligned}
$$

Thus there are no spin- 0 modes in $8_{\mathrm{s}}$ which would be necessary for a massive spin- 2 representation

$$
D\left(E_{0}, 2,8_{\mathrm{s}}\right) \text { of } \mathrm{SO}(3,2) \times \mathrm{SO}(8) .
$$

Fortunately, the general theory of massive modes in Kaluza-Klein theories [18] as well as specific calculations for the internal space $\mathrm{S}_{7}[3,4]$ tell us that the $\mathrm{SO}(8)$ representations which describe massive spin-2 particles correspond to heptospherical harmonics on $\mathrm{S}_{7}$, i.e., to $n$-fold totally symmetric tensor products of $8_{v}$ with Dynkin labels $(n, 0,0,0)$. It is these representations which we take for the Fock vacuum.

The relevant direct products of $(n, 0,0,0)$ with the representations of $(54)$ decompose as

$$
\begin{aligned}
(n, 0,0,0) \times(0,0,0,0)= & (n, 0,0,0), \\
(n, 0,0,0) \times(0,0,0,1)= & (n, 0,0,1) \oplus(n-1,0,1,0), \\
(n, 0,0,0) \times(0,1,0,0)= & (n, 1,0,0)^{-} \oplus(n, 0,0,0)^{*} \\
& \oplus(n-1,0,1,1)^{+} \oplus(n-2,1,0,0)^{-}, \\
(n, 0,0,0) \times(1,0,1,0)= & (n+1,0,1,0) \oplus(n-2,0,0,1) \oplus(n-1,0,1,0)^{*} \\
& \oplus(n-2,1,0,1) \oplus(n, 0,0,1)^{*} \oplus(n-1,1,1,0), \\
(n, 0,0,0) \times(2,0,0,0)= & (n+2,0,0,0) \oplus(n, 0,0,0)^{*} \oplus(n-2,0,0,0) \\
& \oplus(n-2,1,0,0)^{*} \oplus(n-2,2,0,0) \oplus(n, 1,0,0)^{*}, \\
(n, 0,0,0) \times(0,0,2,0)= & (n, 0,2,0) \oplus(n-2,0,0,2) \oplus(n-1,0,1,1)^{*},
\end{aligned}
$$


where, whenever an integer in (56)-(61) becomes negative, the corresponding representation is defined to be absent. The results (56)-(61) were obtained partly by use of the general rules given in [17] and partly by use of the Young tableaux [19]. The latter technique can be employed whenever only one kind of index is involved, and it is useful to note the following correspondence between the Young tableaux and Dynkin labels:

$$
\begin{aligned}
& (n, 0,0,0)=\text { मात }, \\
& (0,1,0,0)=\theta=28, \\
& (0,0,2,0)=\theta_{+}=35_{c},
\end{aligned}
$$

where, in the last line of (62), we have invoked the equivalence of $35_{c}$ and the self-dual antisymmetric four-index tensor with vectorial indices. The decompositions (58), (60) and (61) are then immediate consequences of the general rules for multiplying the Young-tableaux. More importantly, all of (56)-(61) can be checked by verifying the following two sum rules $\{17\}$. If the product of two irreducible representations $\mathbf{R}_{1}$ and $\mathbf{R}_{2}$ decomposes into irreducible representations $\mathbf{R}_{3}, \ldots, \mathbf{R}_{N}$ according to

$$
\mathrm{R}_{1} \times \mathbf{R}_{2}=\bigoplus_{i=3}^{N} \mathrm{R}_{i}
$$

then

$$
\begin{gathered}
D\left(\mathbf{R}_{1}\right) D\left(\mathbf{R}_{2}\right)=\sum_{i=3}^{N} D\left(\mathbf{R}_{i}\right), \\
l\left(\mathbf{R}_{1}\right) D\left(\mathbf{R}_{2}\right)+l\left(\mathbf{R}_{2}\right) D\left(\mathrm{R}_{1}\right)=\sum_{i=3}^{N} l\left(\mathbf{R}_{i}\right),
\end{gathered}
$$

where $D(\mathrm{R})$ denotes the dimension and $l(\mathrm{R})$ the index of the representation $\mathrm{R}$ [17]. Now, for the irreducible representation $\left(a_{1}, a_{2}, a_{3}, a_{4}\right)$ of $\operatorname{SO}(8)$, these two quantities are given by

$$
\begin{aligned}
D\left(a_{1}, a_{2}, a_{3}, a_{4}\right)= & \left(1+a_{1}\right)\left(1+a_{2}\right)\left(1+a_{3}\right)\left(1+a_{4}\right) \\
& \times\left(1+\frac{1}{2}\left[a_{1}+a_{2}\right]\right)\left(1+\frac{1}{2}\left[a_{2}+a_{3}\right]\right)\left(1+\frac{1}{2}\left[a_{2}+a_{4}\right]\right) \\
& \times\left(1+\frac{1}{3}\left[a_{1}+a_{2}+a_{3}\right]\right)\left(1+\frac{1}{3}\left[a_{1}+a_{2}+a_{4}\right]\right) \\
& \times\left(1+\frac{1}{3}\left[a_{2}+a_{3}+a_{4}\right]\right) \\
& \times\left(1+\frac{1}{4}\left[a_{1}+a_{2}+a_{3}+a_{4}\right]\right)\left(1+\frac{1}{5}\left[a_{1}+2 a_{2}+a_{3}+a_{4}\right]\right),
\end{aligned}
$$




$$
\begin{aligned}
l\left(a_{1}, a_{2}, a_{3}, a_{4}\right)= & \frac{1}{28} D\left(a_{1}, a_{2}, a_{3}, a_{4}\right)\left\{a_{1}\left(a_{1}+a_{2}+\frac{1}{2}\left[a_{3}+a_{4}\right]+6\right)\right. \\
& +a_{2}\left(a_{1}+2 a_{2}+a_{3}+a_{4}+10\right)+a_{3}\left[\frac{1}{2}\left(a_{1}+a_{4}\right)+a_{2}+a_{3}+6\right] \\
& \left.+a_{4}\left[\frac{1}{2}\left(a_{1}+a_{3}\right)+a_{2}+a_{4}+6\right]\right\} .
\end{aligned}
$$

Formulae (65) and (67) can be derived with the help of the formulae and tables of [17]. Inserting the various representation labels of (56)-(61) into (66) and (67), one verifies that (64) and (65) are indeed satisfied ${ }^{\star}$.

A final test for the validity of (56)-(61) is provided by the relation

$$
c\left(\mathrm{R}_{1}\right)+c\left(\mathrm{R}_{2}\right)=c\left(\mathrm{R}_{i}\right), \quad i=3, \ldots, N
$$

for the congruence classes $c(\mathrm{R})$ [20]. For $\mathrm{SO}(8), c(\mathrm{R})$ is a two-component vector defined by [20]

$$
c\left(a_{1}, a_{2}, a_{3}, a_{4}\right)=\left(\left(a_{3}+a_{4}\right) \bmod 2,\left(a_{1}+a_{3}\right) \bmod 2\right) .
$$

The results (56)-(61) pass this test, too.

It is gratifying that (56)-(61) contain precisely the required number of helicity $1, \frac{1}{2}$ and 0 states to give masses to the spin-2, spin- $\frac{3}{2}$ and spin- 1 states. These Higgs states have been marked by an asterisk in (56)-(61). Removal of the Higgs states leaves us with the following table for the excited modes on $\mathrm{S}_{7}$ and their Dynkin labels:

$$
\begin{array}{ll}
\operatorname{spin} 2: & (n, 0,0,0), \\
\operatorname{spin} \frac{3}{2}: & (n, 0,0,1) \oplus(n-1,0,1,0), \\
\operatorname{spin} 1: & (n, 1,0,0)^{-} \oplus(n-1,0,1,1)^{+} \oplus(n-2,1,0,0)^{-}, \\
\operatorname{spin} \frac{1}{2}: & (n+1,0,1,0) \oplus(n-1,1,1,0) \oplus(n-2,1,0,1) \oplus(n-2,0,0,1), \\
\operatorname{spin} 0^{+}: & (n+2,0,0,0) \oplus(n-2,2,0,0) \oplus(n-2,0,0,0), \\
\operatorname{spin} 0^{-}: & (n, 0,2,0) \oplus(n-2,0,0,2) .
\end{array}
$$

In principle, (69) contains the solution to the problem of finding all higher modes on $\mathrm{S}_{7}$, but we emphasize once more that a complete proof is still lacking. In particular, the associated energy labels (or mass eigenvalues) are not completely known.

* We are grateful to C.B. Lang for helping us with an algebraic computer program to facilitate this calculation. 
One of us (H.N.) acknowledges the kind hospitality of MIT Department of Mathematics, where most of the research described here was performed. The research was supported by US National Science Foundation grant PHY 81-07394.

\section{Note added}

After this work was completed, we learned that the full bosonic mass spectrum of the round $S_{7}$ compactification of 11-dimensional supergravity has been very recently calculated by Biran, Casher, Englert, Rooman and Spindel, Phys. Lett. 134B (1984) 179. In the region of overlap the results agree with those of sect. 10.

\section{Note added in proof}

As was pointed out at the end of sect. 9, the case $J=\frac{1}{2}$ requires separate study. From (50), one would conclude that the corresponding $N=2$ multiplet contains the representations $D\left(\frac{1}{2}, 0, \frac{1}{2}\right), D\left(1, \frac{1}{2}, \frac{1}{2}\right)$ and $D\left(\frac{3}{2}, 0, \frac{1}{2}\right)$. However, the last representation is, in fact, absent as one can easily verify by inserting the values $E_{0}=J=\frac{1}{2}$ and $s=0$ into (25) and (39). Therefore, the $N=2$ singleton representation is

$$
D\left(\frac{1}{2}, 0, \frac{1}{2}\right) \oplus D\left(1, \frac{1}{2}, \frac{1}{2}\right) .
$$

Obviously, it can not be obtained from the general Higgs procedure, which is consistent with the fact that these states have no proper Poincare limit [21]. We have since learnt that, for $N=8$ as well there exists a singleton representation given by ([22] and second reference in [8])

$$
D\left(\frac{1}{2}, 0,8_{\mathrm{c}}\right) \oplus D\left(1, \frac{1}{2}, 8_{\mathrm{v}}\right) .
$$

Hence, the singleton representations correspond to extreme cases of multiplet shortening.

\section{References}

[1] E. Cremmer, B. Julia and J. Scherk, Phys. Lett. 76B (1978) 409

[2] P.G.O. Freund and M.A. Rubin, Phys. Lett. 94B (1980) 233

[3] M.J. Duff and C.N. Pope, in Supergravity '82, eds. S. Ferrara, J.G. Taylor and P. von Nieuwenhuizen (World Scientific)

[4] B. Biran, B. deWit, F. Englert and H. Nicolai, Phys. Lett. 124B (1983) 45

[5] A. Salam and J. Strathdee, Nucl. Phys. B80 (1974) 499;

E. Witten and D. Olive, Phys. Lett. 78B (1978) 97;

D.Z. Freedman, in Recent developments in gravitation, (Cargèse 1978) eds. M. Levy and S. Deser (Plenum, 1979);

M.F. Sohnius, Nucl. Phys. B138 (1978) 109;

S. Ferrara, C.A. Savoy and B. Zumino, Phys, Lett. 100B (1981) 393

[6] W. Heidenreich, Phys. Lett. 110B (1982) 461 
[7] V.G. Kac, Advances in Math. 261 (1977) 8; Commun. in Alg. 5(8) (1977) 889

[8] I. Bars and M. Günaydin, Comm. Math. Phys., to appear;

M. Günaydin, in Group theoretical methods in physics, Istanbul, Turkey, 1982; Lecture notes in physics 180 (Springer)

[9] C. Fronsdal, Phys. Rev. D12 (1975) 3819

[10] B.W. Keck, J. Phys. A. Math. Gen. 8 (1975) 1819

[11] E.A. Ivanov and A.S. Sorin, J. Phys. A. Math. Gen. 13 (1980) 1159

[12] P. Breitenlohner and D.Z. Freedman, Ann. of Phys. 144 (1982) 249

[13] J. Weitsman, MIT Bachelor Thesis, 1983 (unpublished)

[14] L. Biedenharn and H.V. Van Dam, Quantum theory of angular momentum (Academic Press, 1965)

[15] R. D'Auria and P. Fré, Phys. Lett. 121B (1983) 141

[16] M.J. Duff, B.E.W. Nilsson and C.N. Pope, Phys. Rev. Lett. 50 (1983) 2043

[17] R. Slansky, Phys. Reports 79 (1981) 1

[18] A. Salam and J. Strathdee, Ann. of Phys. 141 (1982) 316

[19] M. Hammermesh, Group theory and its applications to physical problems (Addison-Wesley, 1962)

[20] W. McKay and J. Patera, Tables of dimensions, indices, and branching rules for representations of simple algebras (Dekker, New York, 1981)

[21] M. Flato and C. Fronsdal, Phys. Lett. 97B (1980) 236

[22] E. Sergin, private communication 